\title{
Long-term electrophysiological activity and pharmacological response of a human induced pluripotent stem cell-derived neuron and astrocyte co-culture
}

\author{
A. Odawara ${ }^{1}$, Y. Saito ${ }^{2}$, A.H. Alhebshi ${ }^{1}$, M. Gotoh ${ }^{1,2}$, I. Suzuki $^{1,2^{*}}$ \\ ${ }^{1}$ Graduate School of Bionics, ${ }^{2}$ School of Bioscience and Biotechnology, Tokyo University of \\ Technology, 1404-1Katakura, Hachioji, Tokyo 192-0982, Japan
}

\section{*Corresponding author:}

Ikuro Suzuki

Tel: +81-42-637-2698

Fax: +81-42-637-2698

E-mail address: isuzuki@stf.teu.ac.jp

Abbreviations:

${ }^{1} \mathrm{CNQX}^{2}{ }^{2} \mathrm{DIV},{ }^{3}$ hiPSC, ${ }^{4}$ MEA, ${ }^{5}$ PBS

1 6-cyano-7-nitroquinoxaline-2,3-dione

2 days in vitro

3 human induced pluripotent stem cell

4 multi-electrode array

5 phosphate-buffered saline 


\begin{abstract}
Human induced pluripotent stem cell (hiPSC)-derived neurons may be effectively used for drug discovery and cell-based therapy. However, the immaturity of cultured human iPSC-derived neurons and the lack of established functional evaluation methods are problematic. We here used a multi-electrode array (MEA) system to investigate the effects of the co-culture of rat astrocytes with hiPSC-derived neurons on the long-term culture, spontaneous firing activity, and drug responsiveness effects. The co-culture facilitated the long-term culture of hiPSC-derived neurons for $>3$ months and long-term spontaneous firing activity was also observed. After $>3$ months of culture, we observed synchronous burst firing activity due to synapse transmission within neuronal networks. Compared with rat neurons, hiPSC-derived neurons required longer time to mature functionally. Furthermore, addition of the synapse antagonists bicuculline and 6-cyano-7-nitroquinoxaline-2,3-dione induced significant changes in the firing rate. In conclusion, we used a MEA system to demonstrate that the co-culture of hiPSC-derived neurons with rat astrocytes is an effective method for studying the function of human neuronal cells, which could be used for drug screening.
\end{abstract}

\title{
Keywords:
}

astrocyte co-culture, human induced pluripotent stem cell-derived neurons, long-term measurement, multi-electrode array, pharmacological effects 


\section{Introduction}

Human induced pluripotent stem cells (hiPSCs) differentiation techniques and culture methods are important evaluation models and an alternative to animal testing for drug discovery screening, toxicity testing, and elucidating disease mechanisms [1-3]. Neuronal cells can be generated from hiPSCs, providing a very important alternative to studies of humans and model organisms, to facilitate a better understanding of the mechanisms of neurological diseases and identifying novel therapeutic avenues $[4,5]$. However, hiPSC-derived neurons cultured using traditional two-dimensional culture methods lack adequate maturation, and evaluation methods focusing on the function of cultured hiPSC-derived neurons have not been established. Additionally, using glial cell humoral factor in cultured neuronal cells increases the number of living cells [6, 7]. We previously demonstrated that achieving long-term single cell culture is possible by adding glial cell humoral factor to rat hippocampal neurons [8]. Additionally, synapse maturation was detected in the presence of physical contact with astrocytes [9]. Furthermore, results suggest that synaptic transmission between presynaptic, postsynaptic, and glial cells was enhanced by astrocytes in neuronal networks [10-12], which could be a very important step in mature cultured hiPSC-derived neuron production. A well-known traditional method for measuring the function of cultured neurons is the patch clamp method using a glass micropipette, used to analyze the function of hiPSC-derived neurons [13]. However, this method is invasive and not suitable for long-term measurement. In contrast, using a multi-electrode array (MEA) system facilitates non-invasive, real-time, multi-point measurement of the activity of cultured neurons $[6,14-16]$. Therefore, this may be a suitable method for studying the function of hiPSC-derived neurons. The MEA system has been used to evaluate the plasticity of rat and mouse neuronal networks [15], neuronal diseases [17], and drug responsiveness. A study 
showed that the MEA system is useful for toxicity evaluation and other analytical methods with hiPSC-derived cardiomyocytes [18-20]. Therefore, the MEA system could be useful for studying hiPSC-derived neurons.

Here, we used the MEA system to investigate the functional effects of co-culturing rat astrocytes with hiPSC-derived neurons on their long-term spontaneous activity and drug responsiveness.

\section{Materials and Methods}

\subsection{Planar MEA chip}

To evaluate the long-term culture of hiPSC-derived neurons, we used a planar MEA measurement system (Alpha Med Scientific, Japan). The MEA chips were produced on a glass slide, comprising 64 electrodes $(50 \times 50 \mu \mathrm{m})$ with $150-\mu \mathrm{m}$ spacing in an $8 \times 8$ grid arrangement (MED-P515A; Alpha Med Science). Each recording terminal surface was coated with Pt/Pt-black to reduce impedance.

\section{2. hiSPC-derived neurons}

hiPSC-derived neurons [iCell neurons; Cellular Dynamics International (CDI) Inc., USA] were cultured on MEA chips. The MEA chips were coated with $0.05 \%$ polyethylenimine solution (Sigma-Aldrich) for $1 \mathrm{~h}$ at room temperature, washed four times with sterilized water, and left to dry overnight. Next, $5 \mu \mathrm{g} / \mathrm{ml}$ of laminin solution was added, and the chips were incubated at $37^{\circ} \mathrm{C}$ in a $5 \% \mathrm{CO}_{2} / 95 \%$ air atmosphere. On the day that culture commenced, the laminin solution was removed and a $\varphi 3.4-\mathrm{mm}$ glass ring was placed in the middle of the probes where the electrodes were located. The cells $(90 \mu \mathrm{l})$ were seeded 
inside the rings (density, $1.3 \times 10^{6}$ cells $/ \mathrm{ml}$ ) and incubated for $1 \mathrm{~h}$. Culture medium was applied around the rings and were removed carefully. The cultures were grown at $37^{\circ} \mathrm{C}$ in a $5 \% \mathrm{CO}_{2} / 95 \%$ air atmosphere.

\subsection{Humoral factor derived from rat astrocytes and rat astrocyte co-culture}

iCell neuron maintenance medium (NRM-100-121-001, CDI, Inc., USA) was supplemented with iCell neuron medium supplement (NRM-100-031-001, CDI, Inc., USA) and $100 \mathrm{U} / \mathrm{ml}$ penicillin/streptomycin (Invitrogen). To evaluate the pharmacological response and long-term spontaneous activity characteristics, we tested iCell neurons in three different culture conditions: (i) conventional culture conditions using samples with maintenance medium culture; (ii) conventional culture conditions with the addition of rat astrocyte-derived humoral factor; and (iii) with the addition of rat astrocyte-derived humoral factor and rat astrocytes.

To obtain the rat astrocytes, we cultured rat primary hippocampal neurons for one month in culture medium (Neurobasal medium) supplemented with $2 \%$ v/v B27 supplement, $10 \%$ v/v fetal bovine serum, $100 \mathrm{U} / \mathrm{ml}$ penicillin/streptomycin (all from Invitrogen), and $0.074 \mathrm{mg} / \mathrm{ml}$ L-glutamine (Sigma). The rat astrocytes were seeded (density, $1 \times 10^{3}$ cells/MEA chip) and co-cultured with hiPSC-derived neurons. The humoral factor was obtained from the same culture medium used to produce the astrocytes.

\subsection{Immunocytochemistry}

The samples were fixed in $4 \%$ paraformaldehyde in phosphate-buffered saline (PBS) on ice $\left(4^{\circ} \mathrm{C}\right)$ for $10 \mathrm{~min}$, followed by methanol on ice $\left(-20^{\circ} \mathrm{C}\right)$ for $10 \mathrm{~min}$. The fixed cells were incubated with $0.2 \%$ Triton X-100 in PBS for $5 \mathrm{~min}$, followed by preblock buffer $(0.05 \%$ 
Triton-X and $5 \%$ goat serum in PBS) at $4^{\circ} \mathrm{C}$ for $1 \mathrm{~h}$, and finally in preblock buffer containing a specific primary antibody at $4^{\circ} \mathrm{C}$ for $24 \mathrm{~h}$. The primary antibodies used were rabbit anti-GFAP (Millipore) for the specific labeling of astrocytes, mouse anti- $\beta$-tubulin III (Sigma-Aldrich) for the specific labeling of neurons, and rabbit anti-synaptophysine for the specific labeling of presynapses. All antibodies were dissolved at 1:1000 in preblock buffer. Secondary antibodies (anti-mouse 488 Alexa Fluor and anti-rabbit 546 Alexa Fluor, Molecular Probes; 1:1000 in preblock buffer) and $1 \mu \mathrm{g} / \mathrm{ml}$ Hoechst 33258 were used for nuclear labeling, which were applied for $1 \mathrm{~h}$ at room temperature. The stained cultures were washed twice using preblock buffer (5 min each), rinsed twice with PBS, and viewed using an electron multiplying CCD camera (iXon Ultra 897, Andor). The image intensity was adjusted using ImageJ software.

\subsection{Extracellular recording}

Spontaneous recordings were obtained for $10 \mathrm{~min}$ each week to determine whether the long-term spontaneous firing depended on the culture conditions. The extracellular signals detected by the MEA system were amplified using a 64-channel amplifier (SU-MED64; Alpha MED Science) and stored on a personal computer. A sampling rate of $20 \mathrm{kHz} / \mathrm{channel}$ was used. The cultures were kept in an incubator during the recordings. Firing analyses were performed using Mobius software (Alpha Med Scientific Inc.).

\subsection{Pharmacological tests}

To investigate pharmacological effects, we administered synaptic antagonists to hiPSC-derived neuronal networks on the MEA chips. The GABA $\mathrm{A}_{\mathrm{A}}$ receptor antagonist bicuculline (Sigma-Aldrich) and the AMPA/kainate receptor antagonist 
6-cyano-7-nitroquinoxaline-2,3-dione (CNQX) (Sigma-Aldrich) were used at final concentrations of $10 \mu \mathrm{M}$ and $30 \mu \mathrm{M}$ in the medium, respectively. Spontaneous recordings were obtained for 60 min before treatment with bicuculline or CNQX and again after administration. A 10-min washout was performed in the medium prior to recordings. The cultures were kept in a $\mathrm{CO}_{2}$ incubator between the recordings and washouts. To avoid medium changes and waiting for the effects of drugs, firing analyses were performed during the last 10 min of the 60 -min period after drug administration.

\subsection{Statistical analysis}

All data were expressed as mean \pm standard error, and the statistically significant differences were calculated using a two-tailed paired Student's $t$-test.

\section{Results}

\subsection{Morphological effects on hiPSC-derived neurons and rat astrocyte co-culture}

To investigate the electrophysiological activity of hiPSC-derived neurons, we tested three different culture conditions: (i) hiPSC-derived neurons, (ii) co-culture of hiPSC-derived neurons and rat astrocyte-derived humoral factor, and (iii) co-culture of hiPSC-derived neurons, rat astrocyte-derived humoral factor, and rat astrocytes (Fig. 1A). Figure 1B shows hiPSC-derived neurons cultured on top of a MEA chip (density, $1.3 \times 10^{6}$ cells $/ \mathrm{cm}^{2}$ ). To investigate hiPSC-derived neuron morphology when co-cultured with rat astrocytes and humoral factor, we immunostained the samples at 42 culture days in vitro (DIV). The astrocyte marker GFAP was negative in the first and second culture conditions (Fig. 1C-a), whereas it was positive when hiPSC-derived neurons were co-cultured with rat 
astrocytes, suggesting that culturing rat astrocytes using the MEA probe is possible (Fig. 1C-a). Furthermore, the rat astrocytes and hiPSC-derived neurons were in physical contact, which was demonstrated by the pseudopodial processes (Fig. S1). In Fig. 1C-b, the nucleus was stained with Hoechst 33258. Compared with the condition where we added the humoral factor and rat astrocytes, the conventional condition exhibited fragmentation of the nucleus at 42 culture DIV. Figure 1C-c shows a fluorescent image where we used the neuronal marker $\beta$-tubulin III. The addition of humoral factor and rat astrocytes protected the cells from damage and they had elongated neurites, contrasting with the damaged neurites in the conventional conditions (Fig. 1C-c). Neurite density indicated neuron maturation in the astrocytes co-cultured samples when compared with the humoral factor co-cultured samples. To investigate synapse transmission, we used the presynapse marker synaptophysin and confirmed synapse expression in all culture conditions. Particularly, the expression of high-density synapses was confirmed in the samples co-cultured with astrocytes (Fig. 1C-d).

\subsection{Time course of spontaneous firing in different culture conditions}

Next, we used the MEA system to investigate whether spontaneous firing depended on the culture conditions, where we made measurements once every week. Figure 2A shows representative examples of the spontaneous firing activity patterns, i.e., neurons that continued to fire with a single spike $(0.03-4.2 \mathrm{~Hz})$ (tonic firing), neurons that generated numerous spikes over a period of time (burst firing), and neurons that fired both tonic and burst firings. At 35 culture DIV, we observed that $80 \%$ of firings were tonic firings. Moreover, the action potential signals we obtained had an adequate signal:noise ratio $($ maximum $=12.8)$. 
Figure 2B-a shows the number of electrodes and the firing frequency of the detected spontaneous activity in the form of a matrix. At 7 culture DIV, the conventional culture, humoral factor cultured samples, and humoral factor/astrocyte co-cultured samples had similar firing activity levels. However, the firing activity declined by 14 culture DIV. In the conventional culture, the firing frequency stopped at 28 culture DIV. The firing frequency and electrode signals obtained from the humoral factor cultured samples increased from 28 culture DIV, whereas in the humoral factor/astrocyte co-cultured samples, the firing frequency increased from 21 culture DIV. Figure 2B-b shows the total number of spikes obtained in all culture conditions. In the humoral factor and humoral factor/astrocyte co-cultured samples, the firing frequency increased at 28 and 35 culture DIV, respectively. We also found that the firing frequency of the humoral factor/astrocytes co-cultured samples increased significantly at 7 culture DIV. Figure 2B-c shows the time course for the number of electrodes that detected signals in all culture conditions. Compared with the conventional culture samples, the number of electrodes that detected signals in the humoral factor/astrocytes co-cultured samples was significantly higher at 7, 21, 28, and 35 culture DIV. In the conventional culture samples, no firing activity of $>1 \mathrm{~Hz}$ was detected. However, we detected a $4.2-\mathrm{Hz}$ firing activity in the humoral factor/astrocytes co-cultured samples. Therefore, co-culture with rat astrocytes facilitated long-term spontaneous firing activity in hiPSC-derived neurons.

\subsection{Electrophysiological properties over the course of 3 months in the hiPSC-derived} neurons and rat astrocyte co-culture

To investigate the properties of the long-term co-culture of hiPSC-derived neurons with rat astrocytes, we examined the spontaneous firing activity pattern for $>3$ months. We 
recorded the spontaneous activity for $>120$ days (data not shown). Figure 3A-b shows the time course of the spontaneous firing activity patterns of the 64 electrodes, the number of electrodes that detected signals, and the firing rate. There was an overall decrease in the firing rate between 7 and 21 culture DIV. However, the firing rate increased more than three-fold on 28 culture DIV with a two-fold increase in the number of electrodes that detected signals when compared with 7 culture DIV, whereas the firing rate and the number of electrodes that detected signal remained high until 92 culture DIV (Fig. 3A-a). A cause for the change in the firing activity pattern was neuron migration on the MEA chip surface. At 92 culture DIV, we noticed two changes in the firing pattern. The first change affected burst firing, where Fig. 3B-a shows the time course of the burst firing rate. The total detected firing rate at 92 culture DIV was low, whereas the total detected firing rates were two times higher at 49 and 71 culture DIV. One-third of spikes were of burst firings at 92 culture DIV. In addition, the inter-spike intervals of burst firing at 48,71 , and 92 culture DIV decreased significantly to $117 \pm 2 \mathrm{~ms}, 86 \pm 2 \mathrm{~ms}$, and $57 \pm 1 \mathrm{~ms}$ (mean \pm SE), respectively $(P<0.01$, Fig. 3B-b). The second change we observed was synchronous firing within the networks. At 92 culture DIV, we observed differences in the synchronous firing rates at the electrodes. Figure 3C-a shows synchronous burst firing status at electrodes 22 and 56 (separated by $670 \mu \mathrm{m}$ ). The propagation delay time from $22 \mathrm{ch}$ to $56 \mathrm{ch}$ was $27 \pm 5 \mathrm{~ms}$. This delay time indicates propagation via a chemical synapse.

Moreover, based on the spontaneous activity measurements for the 92 culture DIV samples, we observed different magnitudes and directions in the amplitudes of the mixed waveforms within the burst firings (Fig. 3C-b). This result demonstrated the firing activity of different neurons and the synchronous burst firing due to synapse propagation. These results shows 
that co-culture with astrocytes facilitated more rapid functional maturation and it maintained long-term spontaneous activity in the hiPSC-derived neurons.

\subsection{Measurement of pharmacological effects on hiPSC-derived neurons using a MEA chip}

Next, we used the MEA system to investigate the effects of synapse receptor-related drug administration to clarify the pharmacological response of hiPSC-derived neurons. We applied bicuculline and CNQX to the humoral factor/astrocytes co-cultured samples at 37 and 92 culture DIV, and we observed changes in the spontaneous activity. Figure 4 shows the change in the firing pattern. Bicuculline administration $(10 \mu \mathrm{M})$ increased the firing frequency, whereas CNQX administration $(30 \mu \mathrm{M})$ stopped the firing activity completely, although it was restored after washing the samples. Bicuculline administration increased the amplitude and the firing rate. We detected multiple different waveforms, which we attributed to an increase in the number of firing neurons. No significant differences were observed with the conventional culture samples (data not shown). Figure 4B shows the electrodes that detected signals and the firing frequency of the 64 electrodes. At 37 and 92 culture DIV, the number of electrodes that detected signals and the firing rate increased after bicuculline administration, whereas they decreased after CNQX administration, although the firing rate was restored after washing out the samples. After bicuculline administration, among the treated samples, we detected an increase in the number of electrodes with firing activity (four and 11 more electrodes at 37 and 92 culture DIV, respectively). The firing rate in treated samples increased 2.8- and 2.4-fold at 37 and 92 culture DIV, respectively (Fig. 3C). After CNQX administration, there was a 0.54- and 0.17 -fold reduction in the firing rate at 37 and 92 culture DIV, respectively. 
These results demonstrated that functional ion channel formation was detectable in the co-culture with rat astrocytes, which is typically required for drug applications.

\section{Discussion}

Here, we demonstrated that the co-culture of hiPSC-derived neurons with rat astrocytes allowed the culture to be maintained for $>120$ days with long-term spontaneous activity. We also showed that it is important that hiPSC-derived neurons are in physical contact with rat astrocytes to facilitate a longer culture period and functional maturation. It has been reported previously that rat neurons can achieve synapse maturation when co-cultured with astrocytes $[9,10]$.

The hiPSC-derived neurons cultured with astrocytes produced signals that were detected by more electrodes with increased activity after 3 weeks of culture. The more frequent detection of electrical signals was because of the presence of astrocytes near the electrodes, which was supported by the immunostaining results (Fig. S2). These results suggest that co-culture with rat astrocytes allowed the hiPSC-derived neurons to achieve functional maturation. In our study, we demonstrated that although the animal species were different, the physical contact with astrocytes had beneficial effects. In future, it will be necessary to use human astrocytes in the same way. Furthermore, we detected three types of firing activity patterns, agreeing with those observed in rat. However, at 35 culture DIV, over $80 \%$ of neurons exhibited tonic firing, which is a characteristic of hiPSC-derived neurons. At 7 culture DIV, the results also suggested that the decrease in the number of electrodes that detected signals was related to GABA neuron maturation, where the suppression of the circuit occurred. In future, it will be necessary to clarify the relationship between the firing patterns obtained with different neuron types. At 90 culture DIV, we observed synchronized firing due to chemical synapse 
transmission in the hiPSC-derived neurons. In rat hippocampal and cortical neurons at an equivalent cell density, the synchronized firing activity attributable to chemical synapse transmission occurs after 1 week of culture, whereas synchronized firing is the primary activity after 2 weeks of culture [16,21]. According to our results obtained using hiPSC-derived neurons, they took a longer time to achieve functional maturation. The co-culture with rat astrocytes also facilitated the detection of a major pharmacological response to synapse antagonist treatments. Bicuculline treatment increased the firing rate and the network activity was suppressed via GABA neuron inhibition.

The firing rate increased greatly at 92 culture DIV, including the synchronized burst firing rate, after bicuculline administration, indicating that the cells were pharmacologically responsive and it also validated the long-term culture of the samples. After CNQX administration, we observed that the firing rate took a long period to recover to its original rate after washing the samples. The relationship between CNQX concentration and recovery time requires further investigation. In conclusion, we demonstrated that the co-culture of hiPSC-derived neurons with rat astrocytes facilitated long-term culture and functional measurements were obtained. Moreover, we detected major responses after the addition of synapse antagonist drugs. Therefore, using a MEA system as a measurement method during hiSPC co-culture with rat astrocytes may be beneficial for clarifying the functions of human neuronal circuits and for drug screening applications.

\section{Acknowledgments}

We thank Alpha Med Scientific company for collaborating in this research. This study was supported by the Japan Society for the Promotion of Science Grant Number 24700485 and by 
the Japan Science and Technology Agency Adaptable and Seamless Technology transfer Program (A-step) Grant Number AS251Z02961P.

\section{References}

[1] K. Takahashi, K. Tanabe, M. Ohnuki, et al., Induction of pluripotent stem cells from adult human fibroblasts by defined factors, Cell 131 (2007) 861-872.

[2] M. Bellin, M.C. Marchetto, F.H. Gage, et al., Induced pluripotent stem cells: the new patient?, Nat Rev Mol Cell Biol 13 (2012) 713-726.

[3] A.B. Cherry, G.Q. Daley, Reprogrammed cells for disease modeling and regenerative medicine, Annu Rev Med 64 (2013) 277-290.

[4] M. Peitz, J. Jungverdorben, O. Brustle, Disease-specific iPS cell models in neuroscience, Curr Mol Med 13 (2013) 832-841.

[5] Y. Imaizumi, H. Okano, Modeling human neurological disorders with induced pluripotent stem cells, J Neurochem (2013).

[6] I. Suzuki, K. Yasuda, Detection of tetanus-induced effects in linearly lined-up micropatterned neuronal networks: application of a multi-electrode array chip combined with agarose microstructures, Biochem Biophys Res Commun 356 (2007) 470-475.

[7] T. Fath, Y.D. Ke, P. Gunning, et al., Primary support cultures of hippocampal and substantia nigra neurons, Nat Protoc 4 (2009) 78-85. 
[8] H.W. Muller, W. Seifert, A neurotrophic factor (NTF) released from primary glial cultures supports survival and fiber outgrowth of cultured hippocampal neurons, J Neurosci Res 8 (1982) 195-204.

[9] H. Hama, C. Hara, K. Yamaguchi, et al., PKC signaling mediates global enhancement of excitatory synaptogenesis in neurons triggered by local contact with astrocytes, Neuron 41 (2004) 405-415.

[10] A. Araque, G. Perea, Glial modulation of synaptic transmission in culture, Glia 47 (2004) 241-248.

[11] G. Perea, A. Araque, GLIA modulates synaptic transmission, Brain Res Rev 63 (2010) 93-102.

[12] L.E. Clarke, B.A. Barres, Emerging roles of astrocytes in neural circuit development, Nat Rev Neurosci 14 (2013) 311-321.

[13] A. Haythornthwaite, S. Stoelzle, A. Hasler, et al., Characterizing human ion channels in induced pluripotent stem cell-derived neurons, J Biomol Screen 17 (2012) 1264-1272. [14] J. Pine, Recording action potentials from cultured neurons with extracellular microcircuit electrodes, J Neurosci Methods 2 (1980) 19-31.

[15] Y. Jimbo, T. Tateno, H.P. Robinson, Simultaneous induction of pathway-specific potentiation and depression in networks of cortical neurons, Biophys J 76 (1999) 670-678. [16] A. Odawara, M. Gotoh, I. Suzuki, A three-dimensional neuronal culture technique that controls the direction of neurite elongation and the position of soma to mimic the layered structure of the brain, RSC Advances 3 (2013) 23620-23630.

[17] A.H. Alhebshi, M. Gotoh, I. Suzuki, Thymoquinone protects cultured rat primary neurons against amyloid beta-induced neurotoxicity, Biochem Biophys Res Commun 433 (2013) 362-367. 
[18] L. Guo, R.M. Abrams, J.E. Babiarz, et al., Estimating the risk of drug-induced proarrhythmia using human induced pluripotent stem cell-derived cardiomyocytes, Toxicol Sci 123 (2011) 281-289.

[19] K. Harris, M. Aylott, Y. Cui, et al., Comparison of electrophysiological data from human-induced pluripotent stem cell-derived cardiomyocytes to functional preclinical safety assays, Toxicol Sci 134 (2013) 412-426.

[20] E.G. Navarrete, P. Liang, F. Lan, et al., Screening drug-induced arrhythmia events using human induced pluripotent stem cell-derived cardiomyocytes and low-impedance microelectrode arrays, Circulation 128 (2013) S3-13.

[21] D.A. Wagenaar, J. Pine, S.M. Potter, An extremely rich repertoire of bursting patterns during the development of cortical cultures, BMC Neurosci 7 (2006) 11.

\section{Figure legends}

Fig. 1. (A) Schematic showing the culture of human induced pluripotent stem cell (hiPSC)-derived neurons in different culture condition. (B) hiPSC-derived neurons were cultured on a multi-electrode array (MEA) system. (B-a) Overview of a MEA chip. (B-b) Phase contrast images of cultured hiPSC-derived neurons on 64-electrode arrays. (C) Immunofluorescent analysis of conventional culture (top panel), conventional culture with added astrocyte-derived humoral factor (middle panel), and astrocyte co-culture (bottom panel), after culture for 37 days. (C-a) Immunofluorescence images showing the presence of astrocytes (GFAP) in astrocyte co-culture. (C-b) Images showing the nuclei with Hoechest 33258 marker staining. (C-c) Images showing neurite formation with immunostaining of $\beta$-tubulin III. (C-d) Merged image showing the formation of synapses and cultured 
hiPSC-derived neurons. Red: presynapse marker (syanptophysine). Green: neuronal marker ( $\beta$-tubulin III). Scale bars $=100 \mu \mathrm{m}$.

Fig. 2. Time course of spontaneous firing in different culture conditions. (A) The waveforms represent the three typical spontaneous firing patterns. The pattern detected most frequently was tonic spiking (top panel). The middle and bottom panels show the burst firing and mixed tonic and burst firing patterns, respectively. (B) Grids showing the 64 electrodes where colored electrodes detected signals. Electrodes that detected a higher firing frequency are shown in red. (C) (C-a) Number of total spontaneous spikes detected vs. the time course. (C-b) Numbers of electrodes that detected firing signals in different culture condition vs. time course ( $\mathrm{n}=3, * P<0.05$ vs. conventional neuronal culture).

Fig. 3. Features of long-term spontaneous firing in astrocyte co-culture over the course of 3 months. (A) Time course of spontaneous firing. (a) The $8 \times 8$ grids represent the electrodes that detected signal and the firing frequency after 7, 28, 49, 71, and 92 culture days in vitro (DIV). (b) Numbers of total spontaneous spikes detected vs. the time course, and the number of electrodes that detected firings vs. the time course. (B) Characteristics of burst firing after 3 months. (B-a) Time course of the number of total burst firings per $10 \mathrm{~min}$. The top panel shows typical burst firings at 92 days. (B-b) Time course of the inter-spike interval for burst firings ( $\mathrm{n}>200, * P<0.01$ vs. 49 days). Inter-spike interval indicates the red bar for burst firings, as shown in the top panel. (C) Synchronized burst firings detected at 92 days. (C-a) The waveforms of synchronized spontaneous firings observed by different channels: $22 \mathrm{ch}$ and $56 \mathrm{ch}$ (red double-headed arrows). The waveforms at the bottom represent magnified synchronized firings that propagated burst firings from $22 \mathrm{ch}$ to $56 \mathrm{ch}$ (red arrows). (C-b) 
Synchronized burst firings detected by one electrode. The differences in the direction and amplitude of burst firings indicate signals from different neurons.

Fig. 4. Effects of drugs in hiPSC-derived neurons with astrocyte co-culture. (A) The waveforms represent typical changes in the spontaneous firing patterns detected by the same electrode. The waveforms at the top represent the spontaneous firing pattern before administration. After bicuculline administration $(10 \mu \mathrm{M})$, the firing frequency and amplitude increased (second waveform). After 6-cyano-7-nitroquinoxaline-2,3-dione (CNQX) administration $(30 \mu \mathrm{M})$, spontaneous firing disappeared (third waveform). The waveform at the bottom indicates the recovery of spontaneous firing after washout. (B) The $8 \times 8$ grids represent the electrodes that detected signals and the firing frequency before and after drug administration at 37 and 92 culture days in vitro (DIV). (C) Graph showing the total number of spikes before and after administration at 37 and 92 culture DIV, and the number of electrodes that detected signals. 
Figures

\section{Figure1}

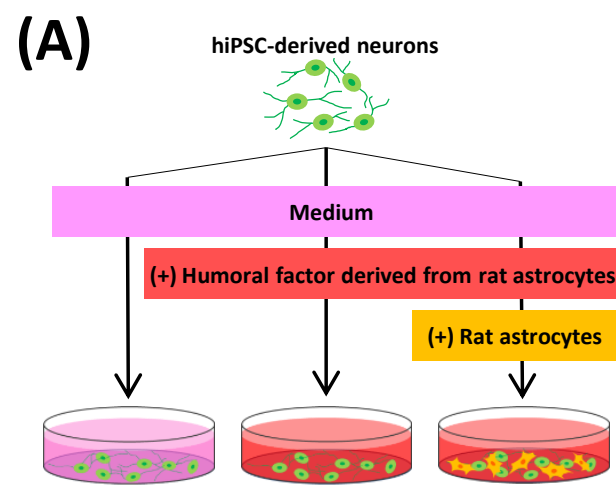

(B)

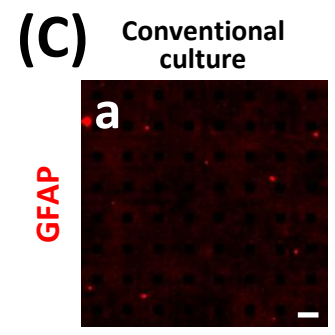

(+) Humoral factor
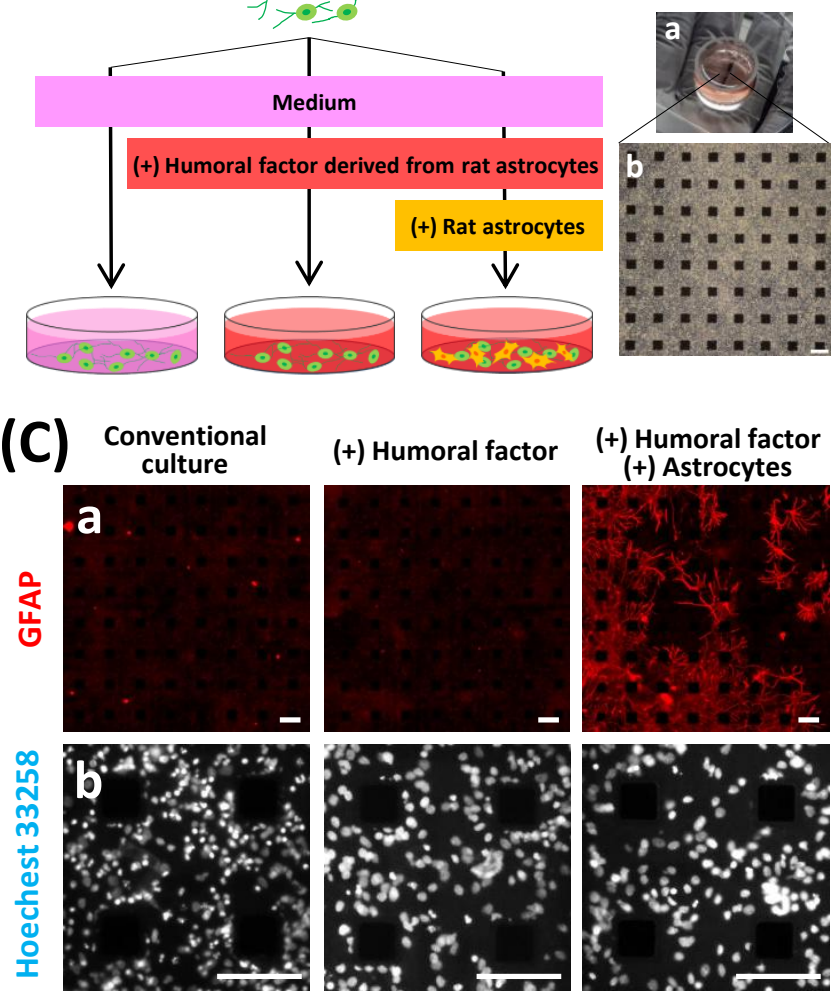

(+) Humoral factor

(+) Astrocytes
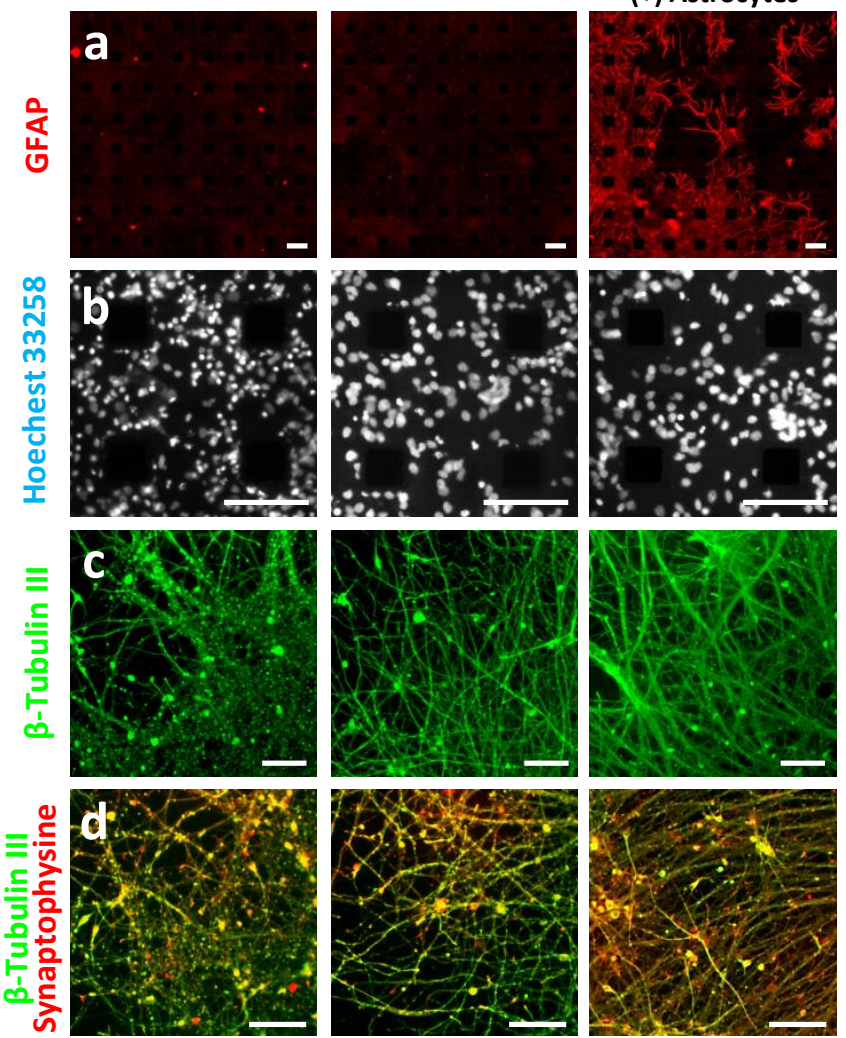
(A)

\section{Tonic firings}

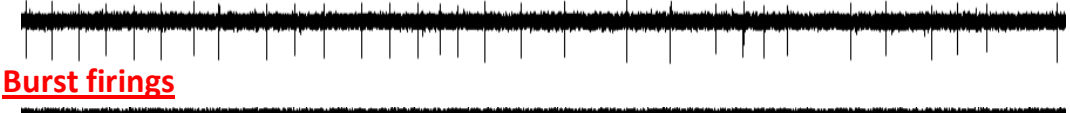

\section{Tonic \& Burst firings}

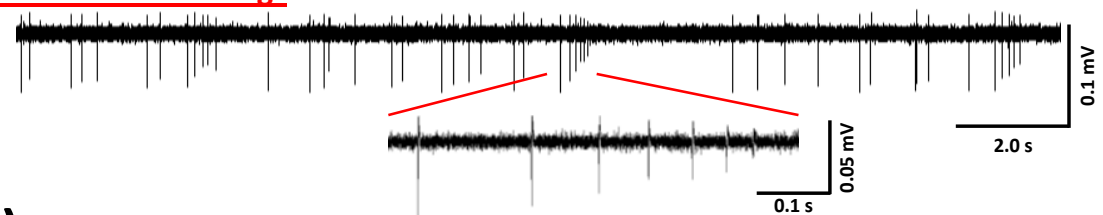

(B)

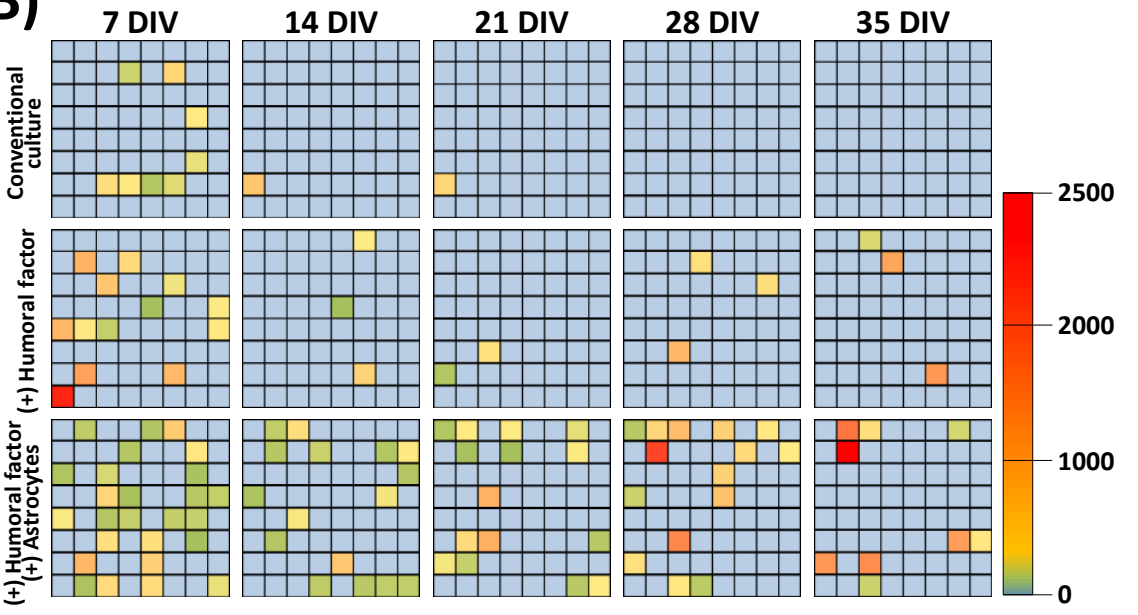

(C) Conventional culture

$$
8000 \text { (+) Humoral factor }
$$
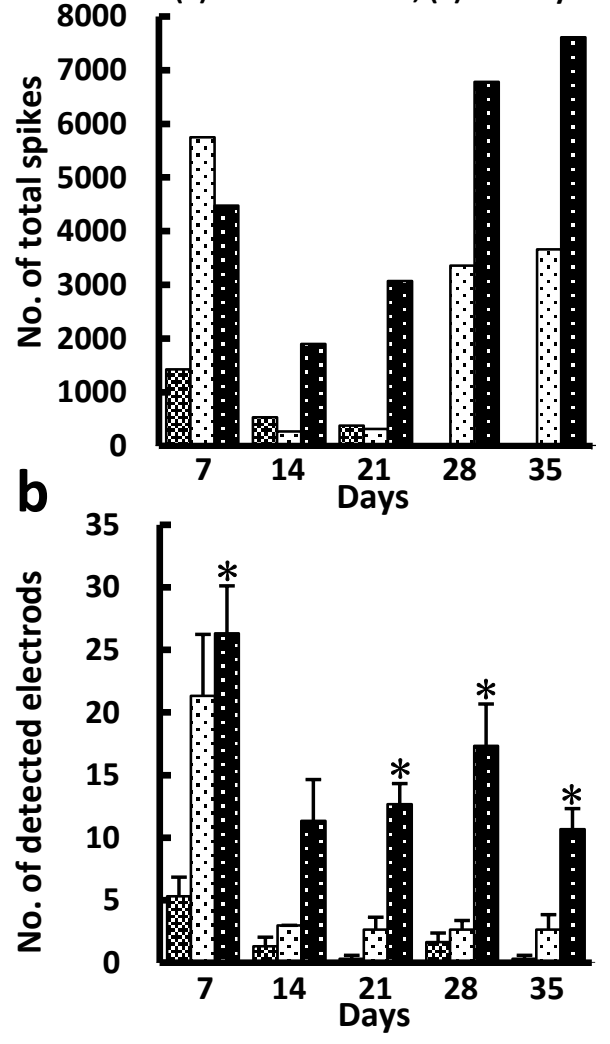
(A) a
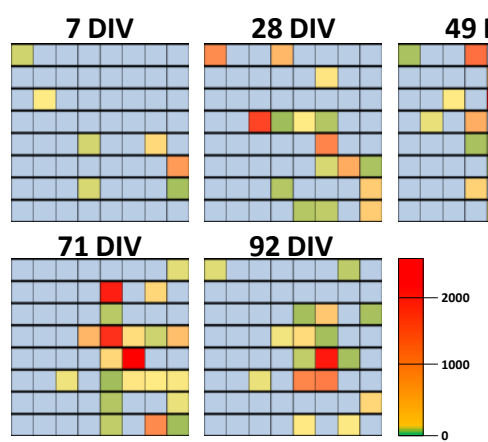

b
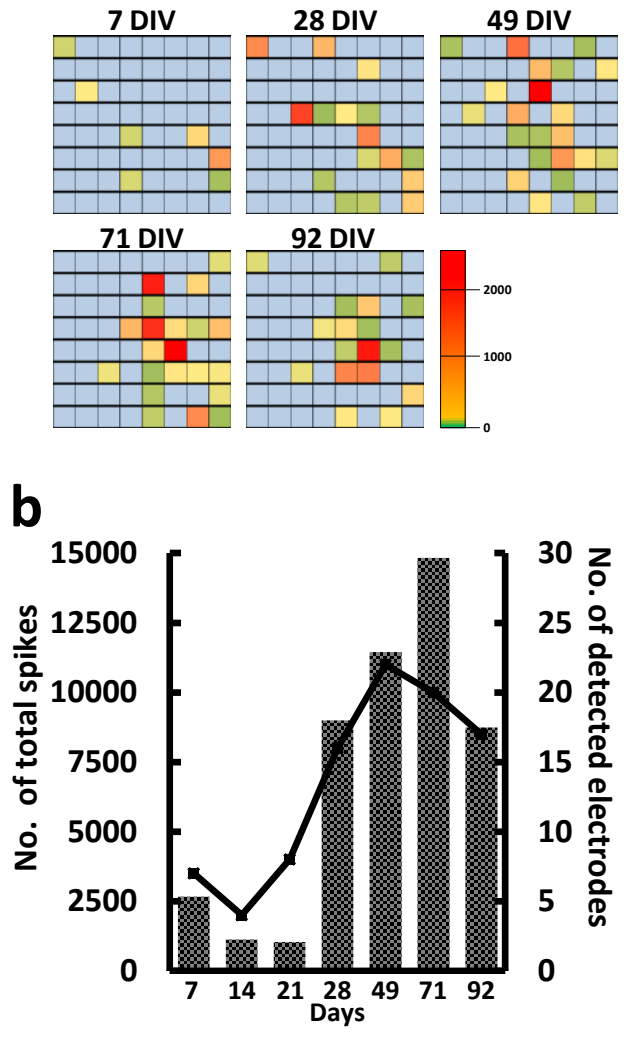

(B) a

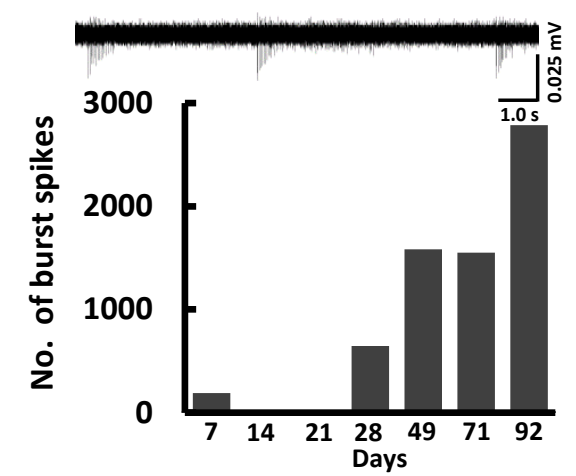

b

b

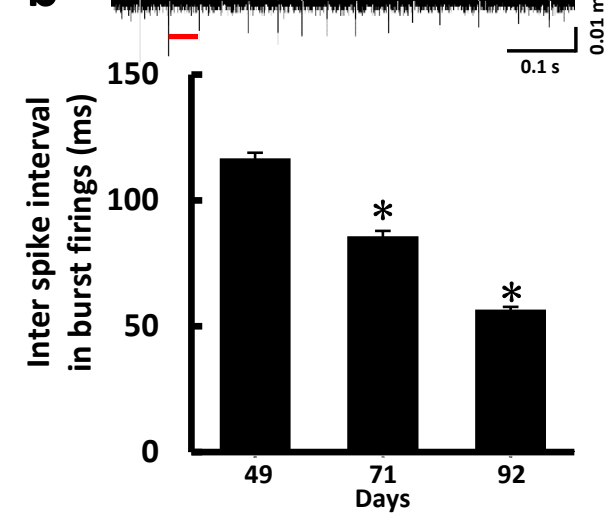

(C) $a$

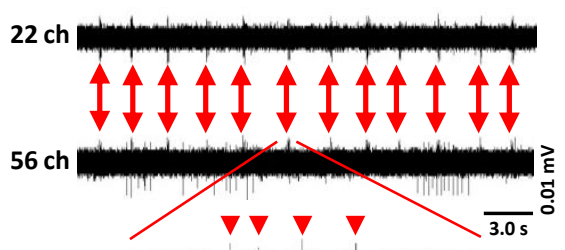

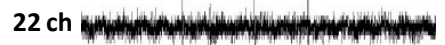

$$
\nabla \nabla \nabla
$$

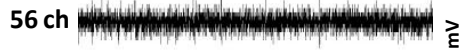

$\mathrm{J}_{50 \mathrm{~ms}} \stackrel{0}{\circ}$

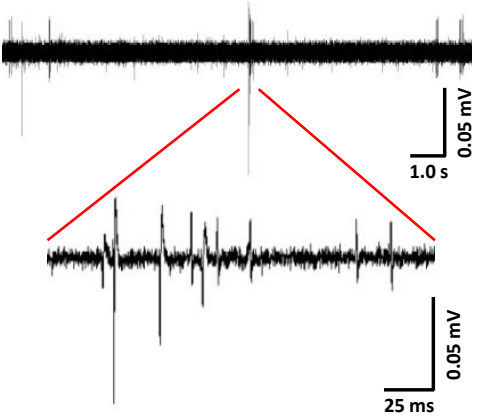


(A)

Before

Bicuculline

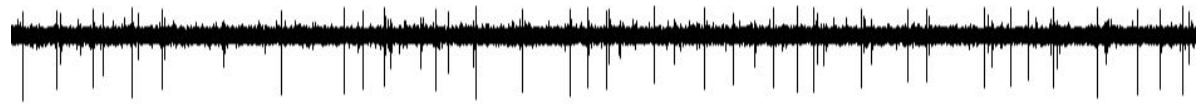

CNQX

After

(B)

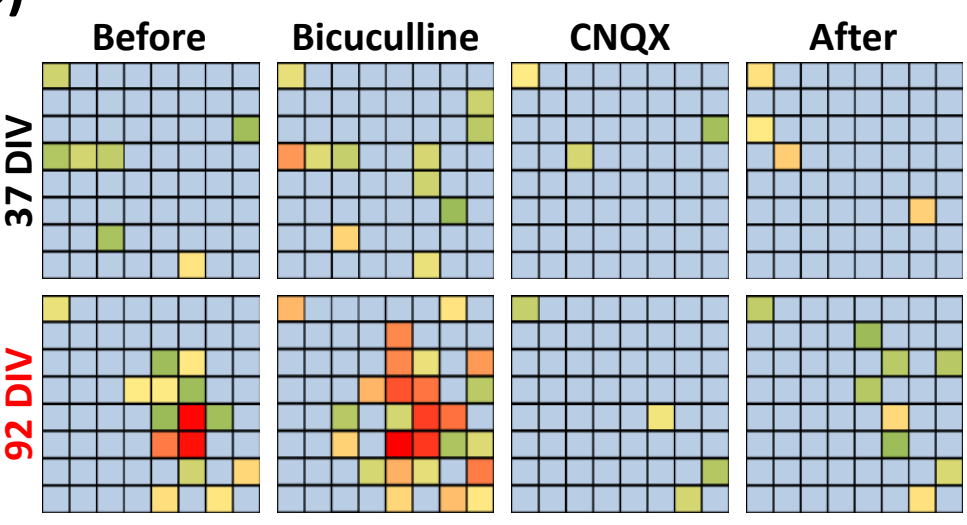

(C)

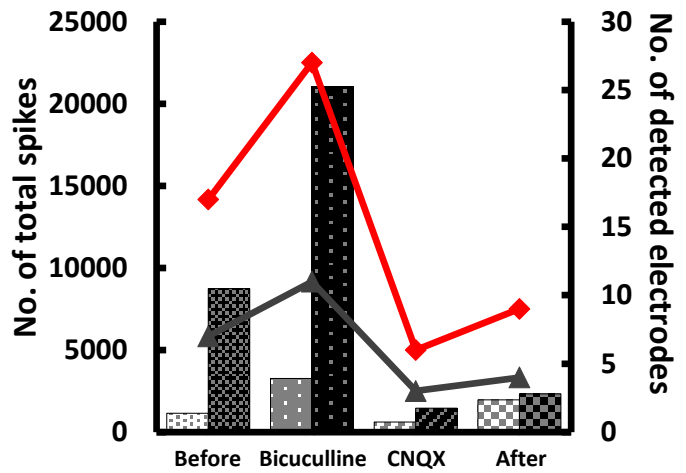

